\title{
MODELO DE SIMULACIÓN PARA PROGRAMAR Y CONTROLAR LOS RECURSOS EN UNA GESTIÓN HOSPITALARIA
}

\begin{abstract}
Alberto Cossa Cabanillas
Resumen

Este trabajo de investigación pretende presentar una base cognoscitiva para la determinación de los productos terminados de las empresas prestadoras de salud. El camino para lograr este cometido se inició con la búsqueda de la forma de cuantificar la necesidad de recursos hospitalarios (horas médico, horas enfermeras, horas cama, entre otros) que requiera un paciente para el tratamiento de su enfermedad. Asimismo, se determinaron y agruparon todos los tratamientos que consumen similares cantidades de estos recursos hospitalarios, mediante el uso de los principios de los grupos de diagnóstico relacionados (DRG); una vez obtenidos los mencionados grupos de tratamientos, se elaboró un modelo de simulación que permita comprobar si estos agrupamientos realizados generan el consumo de recursos dentro de los estándares hospitalarios peruanos; la investigación culminó con la comprobación de estos estándares por parte de las autoridades pertinentes. El gran aporte es la creación del modelo de simulación que permitirá cuantificar la necesidad de recursos de otros agrupamientos y cada agrupamiento puede considerarse como un producto terminado.
\end{abstract}

Palabras clave: diagnosis related group / simulación on line / optimización / lenguaje de simulación AWESIM / sistemas de soporte de decisiones 
Alberto Cossa Cabanillas

\section{Introducción}

Las nuevas tendencias de los mercados pretenden que las empresas abarquen la mayor cantidad de clientes, pertenecientes a distintos estratos sociales y culturas. Todos los clientes tienen necesidades, las cuales generan una diferenciación entre ellos que se contrapone a las diferenciaciones aludidas. Si se entrelazan estas dos diferenciaciones se aprecia el deber de satisfacer las diversas necesidades básicamente en estratos sociales distintos; por ello se requiere un producto que las satisfaga, pero que a la vez cubra las expectativas desde diferentes ópticas sociales. Así, por ejemplo, una plancha satisface la necesidad de tener la ropa planchada; sin embargo, en el mercado podemos encontrar diversos tipos de planchas con diferentes precios disponibles para cada cliente, de acuerdo con su poder adquisitivo. De esta manera, el producto llega a todos los clientes.

Bajo este enfoque, las empresas prestadoras de salud (EPS) deben dar a sus clientes buenos productos que satisfagan sus necesidades según su capacidad. Así, por ejemplo, el producto tratamiento de una apendicitis puede tener diferentes formas de presentación, desde la que demanda los menores costos hasta una de mayores costos. Si se supiera el costo básico de este tratamiento se podría determinar la presentación de menor costo, a la que le acondicionan valores agregados que permitirán el incremento de los costos; de esta manera podemos llegar a la presentación de mayor costo.

Este artículo tiene como principal objetivo presentar un modelo de simulación del funcionamiento hospitalario, el cual permitirá programar y controlar los recursos en una gestión hospitalaria; de esa forma se podrá determinar la presentación del tratamiento de menor costo y a partir de allí fijar las demás presentaciones. Para lograr este cometido, el artículo se ha dividido en cuatro partes: en la primera se describe el sistema real (funcionamiento del hospital), en la segunda se desarrolla un modelo de simulación para el funcionamiento del hospital, en la tercera se hace el análisis de los resultados de la simulación y su validación correspondiente, y en la cuarta parte se presentan las conclusiones y recomendaciones.

\section{El sistema real}

\subsection{El entorno del sistema}

Para usar la metodología de la industria manufacturera en la planificación y control de las empresas de servicio, se debe empezar por tener la nómina de productos terminados y con ellos su lista de materiales (BOM); en el caso del hospital sería la lista de recursos (BOR). Por ello, en el entorno del problema se consideró la determinación de los grupos de diagnóstico relacionados (Diagnosis Related Group - DRG) 
que se usan en el Hospital Nacional Arzobispo Loayza (H. N. Arzobispo Loayza).

Es cierto que el mencionado hospital no usa la metodología de los DRG, que es hasta hoy la mejor herramienta para determinar los productos terminados de un hospital.

La determinación de los DRG existentes en dicho hospital se obtuvo mediante el uso de APG Grouper, una herramienta utilizada en Estados Unidos con el mismo propósito y que fue proporcionada por Codemaster, empresa que elabora software para la codificación de DRG; la herramienta software proporcionada fue APG Grouper en la versión 2.0.

Para usar esta herramienta se utilizaron los datos contenidos en la información dada por la unidad de estadística del hospital, la cual — para que tenga relevancia- abarcó un año y fue capturada de forma aleatoria; fueron 1.020 registros, cada uno correspondiente a un paciente. Una vez obtenido este consolidado se determinó trabajar sobre el DRG 88, que corresponde al 1,8\% del universo de pacientes, por cuanto en esa área se tendría un mejor acceso.

\subsection{Supuestos y descripción del sistema}

Para hacer más comprensible el sistema a simular se consideró conveniente describir cómo se ejecuta el sistema real, y a la vez definir los elementos que en esa ejecución se necesitan. El proceso para hospitalizar, mantener hospitalizado y dar de alta a un paciente se detalla a continuación.

\subsubsection{Términos usados en el modelo}

El comentario dado para cada término no es una definición formal, solo se refiere a su uso dentro del hospital y se consiguió en conversaciones con las personas que laboran en dicho nosocomio.

a) Historia clínica.- Es un documento interno del hospital, donde se muestra la evolución de la enfermedad del paciente. Consta de diferentes hojas: hoja de identificación, hoja de consulta externa, hoja de examen clínico, hoja de notas de evolución, hoja de evaluación subjetiva, hoja de gráfica de controles vitales, hoja de balance hídrico, hoja de tratamiento de enfermería pediatría y hoja de exámenes complementarios.

b) Diagnóstico por imágenes.- Es una herramienta que ayuda al médico a centrar mejor su diagnóstico. Es ejecutado por el Laboratorio de Radiología y Diagnóstico por Imágenes, a pedido del médico especialista. Los encargados de sacar las placas lo hacen a partir de las 15.00 horas. Los diagnósticos por imágenes se realizan al 80\% de pacientes que pertenecen a este DRG, y cada 
paciente necesita entre uno o dos de estos diagnósticos en un aproximado del $50 \%$ cada uno.

c) Exámenes de laboratorio.- Es otra herramienta que ayuda al médico a centrar mejor su diagnóstico. Es ejecutada por el Laboratorio Clínico, a pedido del médico especialista. Los encargados de sacar las muestras lo hacen a partir de las 15.00 horas. Estos exámenes se realizan a todos los pacientes internados y en un número de cuatro por cada paciente; este puede repetir dos o tres veces cada análisis, dependiendo del proceso de recuperación, lo que lleva a incrementar el número de días que debe estar hospitalizado.

d) Procedimientos.- Son las labores que ejecuta el médico especialista o las enfermeras en el paciente. Se clasifican en:

- Terapéuticos. Ayudan a curar al paciente. Son procedimientos terapéuticos la cateterización umbilical, la fototerapia, la oxigenoterapia, la nebulización, la ventiloterapia y la entubación oratraqueal.

- Diagnóstico. Contribuyen a que el médico pueda centrar mejor el diagnóstico. Son procedimientos de diagnóstico el aspirado gástrico, la biopsia, la cateterización vesical, la punción lumbar y la punción de médula ósea.

e) Interconsultas.- Son las visitas de los médicos especialistas en otras áreas (gastroenterología, cardiología, entre otras), que son pedidas por el galeno en el momento de su visita médica, si lo cree necesario. El médico que se encarga de la interconsulta la realiza por la tarde y su duración es de aproximadamente quince minutos. La probabilidad de necesitar interconsultas es del 10\% para los pacientes de la sala A, 20\% para los de la sala B y de 40\% para los de la sala C.

f) Agentes.- Se denominan así a todas las personas y actos involucrados en la atención hospitalaria, desde el paciente hasta las tareas de los médicos y enfermeras.

i. Pacientes: Ingresan a la sala de hospitalización a las 11:00 horas de cada día. Si un paciente que necesita ser hospitalizado llegara en un horario distinto, espera en la sala de emergencia a ser transferido en el horario establecido.

La zona de hospitalización consta de cuatro salas, tres de las cuales han sido divididas por edades y una es para cuidados intensivos. En la primera sala, denominada sala A, son hospitalizados los pacientes menores de dos años. En la segunda sala, sala B, son hospitalizados los pacientes cuyas edades fluctúan entre dos y seis años. En la tercera sala, sala C, son hospitalizados los pacientes con edades entre seis y dieciocho años. En la cuarta sala, denominada unidad de cuidados intensivos UCl, son hospitalizados los pacientes que están en estado crítico. Se debe mencionar que, para esta clasificación de enfermedades (DRG88), el H. N. Arzobispo Loayza solo atiende a pacientes de hasta dieciocho años. Basados en la muestra que se tomó en los quince días de observaciones, el número de pacientes que buscaron internamiento por el DRG88 fueron once; de estos, dos (aprox. 20\%) fueron 
menores de dos años; cinco (aprox. 45\%) tuvieron entre dos y seis años, y los últimos cuatro (aprox. 35\%) tuvieron más de seis años. De los once uno (aprox. 10\%) fue paciente de UCl.

El tiempo de hospitalización de los pacientes, según la estadística obtenida, está entre cuatro y diez días. La probabilidad de que un paciente esté hospitalizado un determinado número de días se presenta en la siguiente tabla:

\begin{tabular}{|l|c|c|c|c|c|c|c|}
\hline Número de días & 4 & 5 & 6 & 7 & 8 & 9 & 10 \\
\hline Probabilidad & 0,10 & 0,70 & 0,06 & 0,05 & 0,04 & 0,03 & 0,02 \\
\hline
\end{tabular}

Tabla 1. Distribución del tiempo de hospitalización de los pacientes

Elaboración propia.

Se consideran dos tipos de pacientes: los nuevos y los antiguos. Los pacientes antiguos son aquellos que por algún motivo, nacimiento, consulta, etcétera, tienen historia clínica en el hospital; este tipo de pacientes constituye el $95 \%$ del total de atendidos. Los pacientes nuevos son los que por primera vez van a ser atendidos en el hospital y representan el $5 \%$ del total.

Todo paciente nuevo o antiguo antes de ocupar la cama pasa por una entrevista con el médico residente. En esta entrevista, que dura entre quince y veinticinco minutos, el paciente es evaluado y se llena la hoja del examen clínico. Una vez terminada, el paciente ocupa su cama. Durante ese día el paciente es medicado de la forma como el médico que recomendó su internamiento lo prescribió.

Durante su estadía en la sala el paciente es controlado por el médico y medicado por la enfermera; si el médico prescribe algún tipo de análisis o pruebas se le hace y será dado de alta cuando este lo crea conveniente. De acuerdo con la política del hospital, las altas normalmente se dan en las tardes.

El día de alta, el paciente recibe la orden respectiva extendida por el médico especialista; si es asegurado va con ella a la oficina de seguros para recibir el formato de alta, y en caso de no ser asegurado va a los laboratorios y a la farmacia para que le entreguen las boletas que debe pagar y luego se dirige a caja para cancelarlas y sale con un tiquete de pago. Ya sea con el formato de alta o con el tiquete de pago, el paciente se retira del hospital; los tiempos que necesita para ejecutar estas tareas no se han puesto en el modelo por considerarlos como fuera del servicio de hospitalización.

ii. Visitas médicas: Una visita médica es el control que el médico hace al paciente diariamente; la realiza un equipo de personas compuesto por: un 
médico especialista (pediatra), dos médicos internos y un médico residente. Para las cuatro salas se han dispuesto tres médicos especialistas, seis médicos internos y dos médicos residentes.

Médico especialista es aquel profesional en medicina que ha concluido y ejerce su especialidad; médico interno es quien ha terminado sus estudios universitarios pero aún no ha obtenido el título de médico, y médico residente es el que ya se ha titulado y está haciendo alguna especialización. Todo médico especialista tiene a su cargo la visita de una sala. Los médicos internos se dividen de dos en dos para acompañar a los médicos especialistas. Los médicos residentes acompañan a un médico especialista en todas sus visitas y en la mitad del número de estas de un segundo médico.

La visita médica a un paciente dura entre trece y diecisiete minutos, y se lleva a cabo entre las ocho y las once de la mañana. Durante esta visita se revisa la historia clínica, se controla el estado del paciente y se toman decisiones a partir de ese estado. Estas pueden ser: pedir algún tipo de análisis, algún tipo de prueba o algún tipo de procedimiento, por citar algunas. Todas estas indicaciones son anotadas en la hoja denominada "Notas de evolución". Asimismo, durante esta visita el médico especialista presenta sus puntos de vista a los otros dos tipos de médicos y absuelve las preguntas que le pudieran hacer.

En la sala UCI puede haber pacientes de cualquier edad, así las visitas a esta sala la harán los médicos, con su respectivo equipo, que tengan pacientes que correspondan a su sala; el proceso dentro de ella es el mismo que en cualquier otra visita médica.

Durante la visita médica, los médicos especialistas pueden prescribir: diagnósticos por imágenes (en un 80\%); exámenes de laboratorio (cuatro exámenes por cada nuevo paciente y al menos dos por paciente durante su estadía); u otros procedimientos de diagnóstico. Los procedimientos que el médico especialista tenga que realizar los hará después de terminada su visita, a no ser que el estado del paciente amerite hacerlo en el momento de esta (solo un 5\%).

iii. Enfermeras: Las enfermeras son profesionales que apoyan las actividades de los médicos y de los pacientes. Para el DRG que estamos analizando, el trabajo de estas profesionales está dividido en dos turnos: el primero inicia sus tareas a las 8.00 horas de cada día y está compuesto por tres enfermeras, dos de ellas trabajan hasta las 20.00 horas, y la otra, la jefa de enfermeras, solo lo hace hasta las 14.00 horas; el segundo grupo inicia sus tareas a las 20.00 horas de cada día y está integrado por dos enfermeras. En el modelo se ha descartado el trabajo de la jefa de enfermeras por ser netamente administrativo. 
Los dos grupos realizan las mismas operaciones en distintos horarios; lo primero que hacen es recibir la información, por parte del grupo saliente, del estado de cada uno de los pacientes internados; esta tarea demanda de un tiempo que está distribuido uniformemente entre uno y dos minutos por paciente.

Su segunda tarea consiste en tomar los síntomas vitales de cada uno de los pacientes, así como administrarles las dosis médicas prescritas por el médico en la historia clínica. Los síntomas vitales son escritos en la hoja de "Evaluación subjetiva" (una hoja por día). Esta tarea demanda un tiempo distribuido uniformemente entre uno y dos minutos por paciente.

La tercera tarea es supervisar el trabajo del personal técnico: cambio de ropa de cama, bañado de pacientes, etcétera, además de apoyar a los facultativos en la visita médica. Esta labor les demanda un tiempo que varía entre 100 y 150 minutos.

La cuarta tarea demanda un tiempo distribuido uniformemente entre siete y diez minutos por paciente. Corresponde al llenado de las demás hojas de la historia clínica: gráfica de controles vitales, tratamiento de enfermería pediatría, balance hídrico, exámenes complementarios. Este llenado se realiza basándose en la hoja de "Evaluación subjetiva", así como en el tratamiento indicado por el médico en la hoja "Notas de evaluación", y de los resultados de los análisis que se reciben del laboratorio de rayos $X$ y del laboratorio clínico.

iv. Técnicos de enfermería: Este personal apoya a las enfermeras en el trabajo directo con el paciente. Sus horarios de trabajo son los mismos que los de las enfermeras y se organizan en dos grupos, el primero trabaja desde las 7.30 horas hasta las 20.00 horas, compuesto por dos técnicos, y el segundo desde las 20.00 horas hasta las 8.00 horas, también conformado por dos técnicos. El trabajo de los técnicos de enfermería se ha omitido en el modelo porque puede determinarse a partir del trabajo de las enfermeras.

v. Otros: Además de los agentes listados, se cuenta también con una profesional en psicología, personal de vigilancia, de limpieza, de nutrición (encargado de gestionar los alimentos de los pacientes), secretaria del Servicio de Pediatría y secretaria del Departamento de Pediatría.

Algunas consideraciones adicionales para la simulación:

- Inicio de la atención: lunes, a las 8.00 horas.

- Inicio de la llegada de los pacientes buscando internarse: lunes, a las 11.00 horas.

- Inicio de las visitas médicas: 8.30 horas de cada día. Se considera que la primera visita para los pacientes internados el lunes (inicio de la simulación) se realiza el martes. 
- Las altas se efectúan todos los días, a las 17.00 horas; la encargada de llevarlas a cabo es la enfermera, pero quien la otorga es el médico en su visita correspondiente. Se consideran a partir del martes, ya que los pacientes ingresaron el lunes.

- Llegada de las enfermeras: 8:00 horas para el primer grupo y a las 20.00 horas para el segundo. Para efectos de esta simulación, se considera que la llegada de las enfermeras recién ocurre el día martes.

\section{El modelo de simulación}

\subsection{Determinación de la data}

Para determinar las duraciones necesarias, las cuales podrían ser constantes o ajustarse a un determinado tipo de distribución en el modelo de simulación, se tomaron muestras (tiempos) directamente del momento en que se hacían las diferentes actividades. Mediante el uso de una prueba piloto se observó que los datos no tenían mucha variación, lo cual fue corroborado por el personal que labora en la sección donde se realizó la investigación; por ello, se eligió una muestra aleatoria concerniente en quince observaciones tomadas para pacientes distintos (cinco por día) en días diferentes (tres días). Con estos tiempos, y con la ayuda del software Stat::Fit, se han definido las distribuciones correspondientes. Como ejemplo del trabajo realizado se muestra la determinación del tiempo usado por un paciente nuevo o antiguo en la entrevista con el residente antes de ocupar una cama.

\begin{tabular}{|c|c|c|c|}
\hline Datos observados & \multicolumn{3}{|l|}{$18,15,25,19,23,18,18,23,17,21,17,22,22,17,24$} \\
\hline Unidad de medida & \multicolumn{3}{|l|}{ Minutos } \\
\hline Resultados de Stat::Fit & $\begin{array}{l}\text { Auto: : Fit Distributions } \\
\text { distribution } \\
\text { Uniform }(15 ; 25) \\
\text { Weibull }(14,1 ; 2,08 ; 6,63) \\
\text { Pearson } 5(4,75 ; 25,2 ; 367) \\
\text { Lognormal }(4,63 ; 2,71 ; 0,197) \\
\text { Extreme Value }(18,5 ; 2,56) \\
\text { Triangular }(14,3 ; 27,2 ; 17) \\
\text { Logistic }(19,8 ; 1,84) \\
\text { Normal }(19,9 ; 2,98) \\
\text { Beta(15;25;1,76;1,76) }\end{array}$ & $\begin{array}{l}\text { rank } \\
89 \\
86,7 \\
85,1 \\
75,9 \\
70,1 \\
53,8 \\
51,8 \\
44,2 \\
34,9\end{array}$ & $\begin{array}{l}\text { acceptance } \\
\text { accept } \\
\text { accept } \\
\text { accept } \\
\text { accept } \\
\text { accept } \\
\text { accept } \\
\text { accept } \\
\text { accept } \\
\text { accept }\end{array}$ \\
\hline Conclusión & \multicolumn{3}{|c|}{$\begin{array}{l}\text { El tiempo usado por un paciente en la entrevista con el residente debe } \\
\text { ser considerado como el valor obtenido de una distribución uniforme } \\
\text { con menor valor } 15 \text { y mayor valor } 25 \text {. }\end{array}$} \\
\hline
\end{tabular}

Tabla 2. Determinación de la distribución del tiempo de entrevista por paciente.

Elaboración propia. 


\subsection{Desarrollo de un modelo de simulación}

El desarrollo del modelo realizado en SLAM System se detalla a continuación.

- Definición de los recursos usados en el modelo. Llegada y hospitalización de los pacientes:

- Se ha considerado a los médicos (residentes, pediatras e internos) como recursos. Se estima que la cantidad de pacientes que llegan a diario buscando hospitalización está distribuida uniformemente entre 35 y 46 personas.

- De los pacientes que llegan diariamente, el 1,8\% está dentro del DRG seleccionado para el estudio. De todos los pacientes que llegan a esta área el $20 \%$ son menores de dos años (tipo 1), el $45 \%$ tiene una edad que fluctúa entre dos y seis años (tipo 2) y el 35\% tiene más de seis años (tipo 3).

- El tiempo de hospitalización de los pacientes fluctúa entre 4 y 10 días, con ciertas probabilidades que no siguen una distribución específica.

- Una vez que el paciente tiene estos datos pasa a la entrevista con el médico residente. Todo paciente que es hospitalizado requiere, dentro del tiempo de estadía, un determinado número de pruebas (diagnóstico de imágenes, pruebas de laboratorio), o algo más especializado o interconsulta.

- Terminada la labor del residente, el paciente ocupará la cama correspondiente según lo indique la necesidad de UCI (10\%) o no, y su edad. Si no necesita la UCl ocupará las salas: A, B o C; y si la necesitara ocupará dicha sala.

- Visita diaria de los médicos: Para las visitas diarias de los médicos se forman los respectivos equipos y cada uno visita la sala correspondiente - A, B o C - , terminado su paseo por esta sala visita, si hubiera, a los pacientes correspondientes de la sala UCl.

- Búsqueda y alta de pacientes: El modelamiento de las altas se ha hecho de la siguiente manera: a partir de las 4:00 p.m. una entidad revisa a los pacientes de cada sala, que en el modelo son entidades dentro de archivos. A los pacientes que se les da de alta son enviados a los modelos correspondientes que permitan recolectar datos para las estadísticas del modelo.

- El trabajo de las enfermeras: Para el trabajo de las enfermeras se crean cuatro entidades, dos para cada turno; hay que recordar que el trabajo de la enfermera jefe no se modela por ser netamente administrativo. A cada una de ellas se les asigna un número: si es del turno de la mañana será del tipo 1 y si es del turno de la tarde será del tipo 2.

Enseguida se registra el momento en que las enfermeras comienzan su turno de trabajo, para posteriores estadísticas. Como son dos enfermeras, cada una de ellas se encarga de la mitad de pacientes que existan en las salas. 


\subsection{Recolección de datos para estadísticas}

Finalmente, se presenta la sección donde se recoge la información que servirá para validar el modelo. Esto se logra mediante el uso de variables. A continuación se muestran las partes del modelo de simulación correspondiente a definición de los recursos usados en el modelo: Llegada y hospitalización de los pacientes y Visita diaria de los médicos.
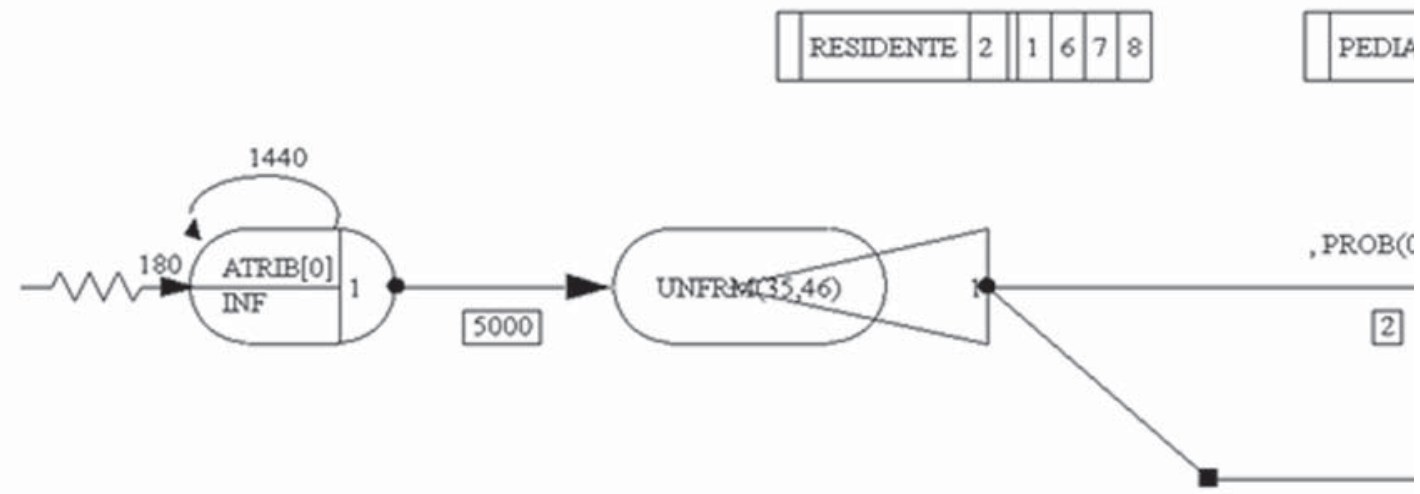

3

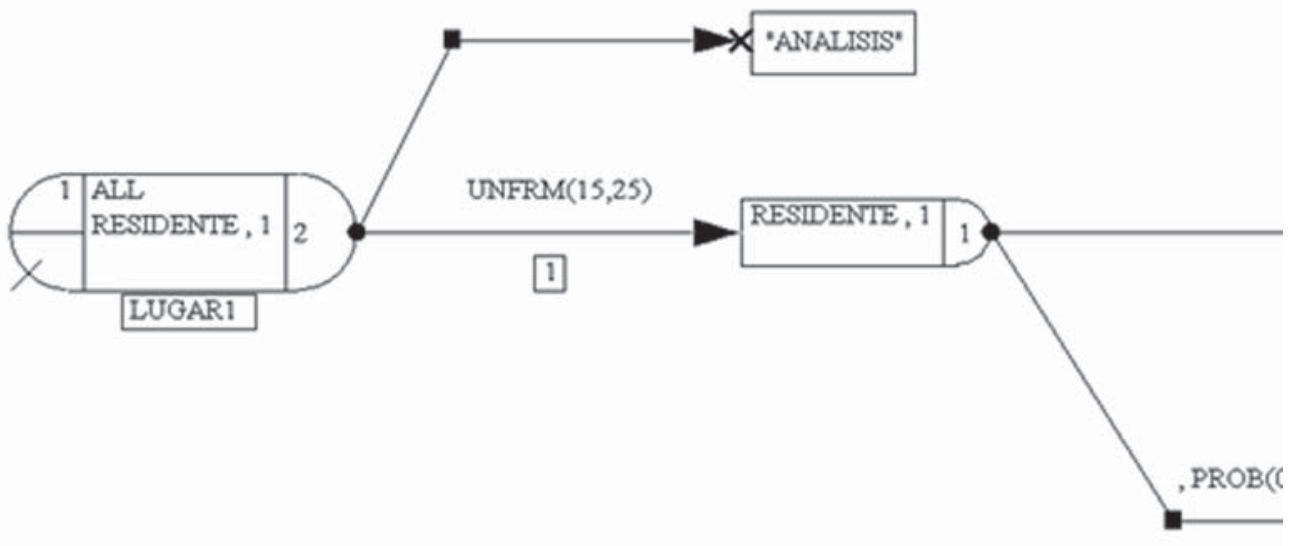

Figura 1. Definición de los recursos usados en el modelo: Llegada y hospitalización de los pacientes. Elaboración propia. 

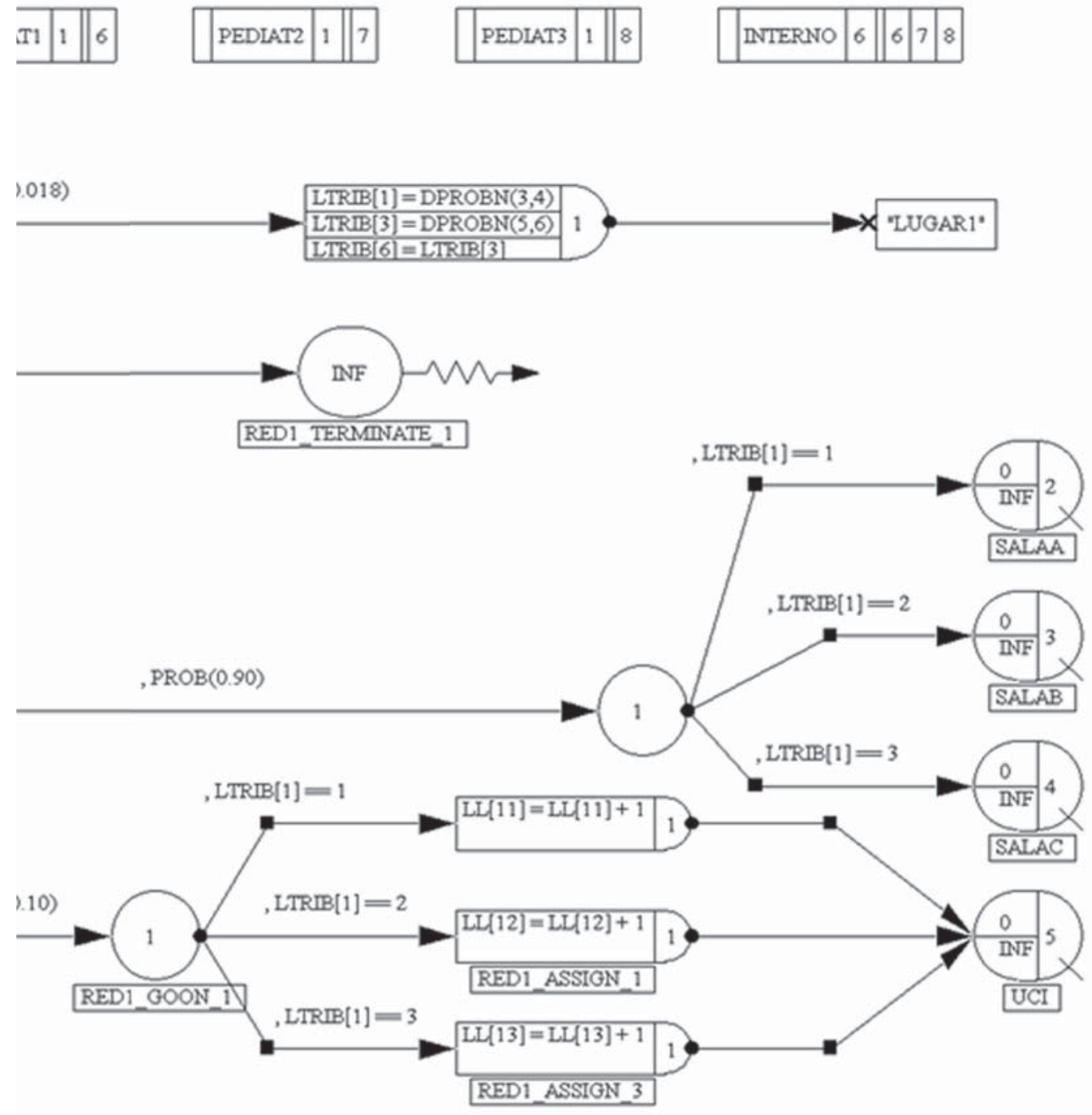
Alberto Cossa Cabanillas

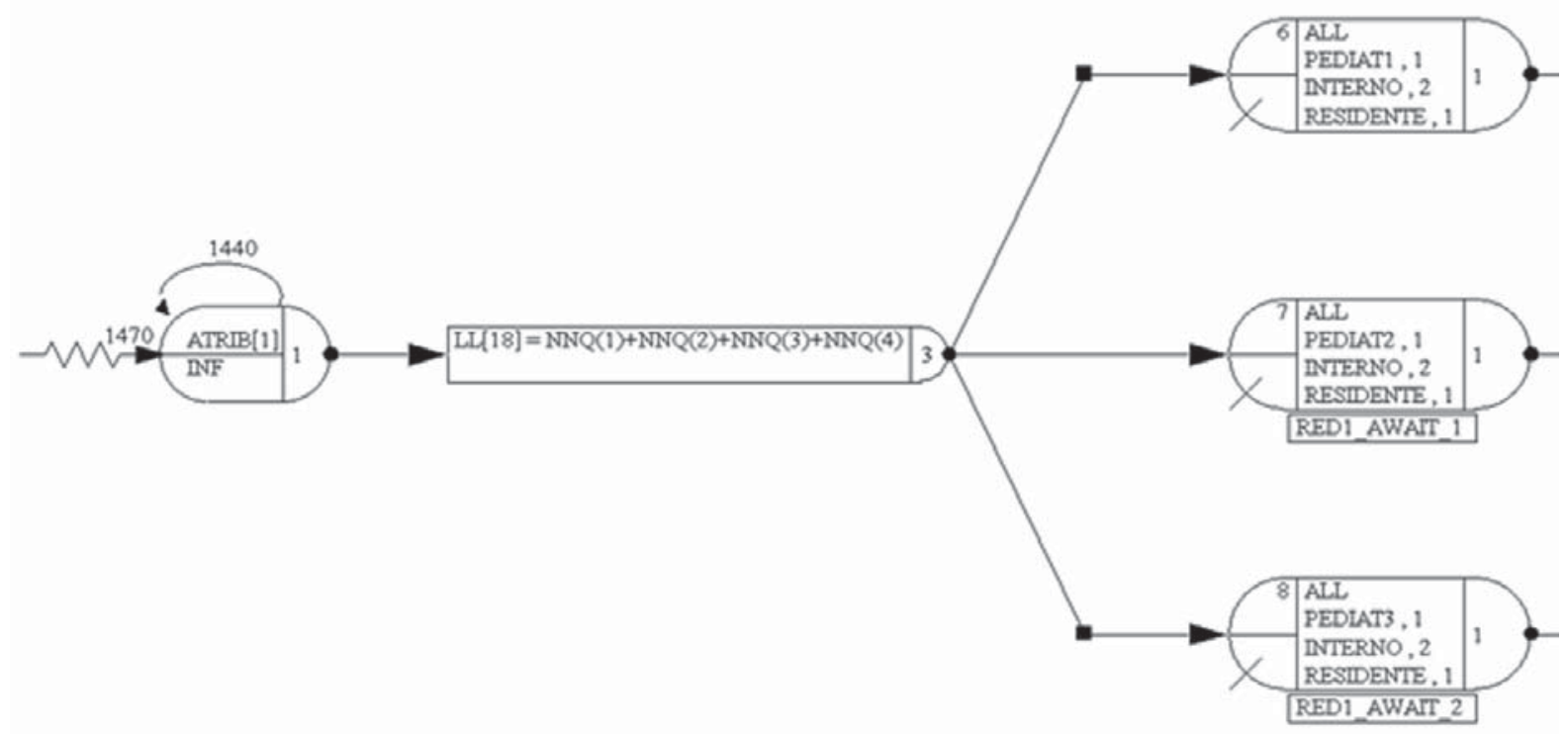

Figura 2. Visita diaria de los médicos: Generalidades.

Elaboración propia. 

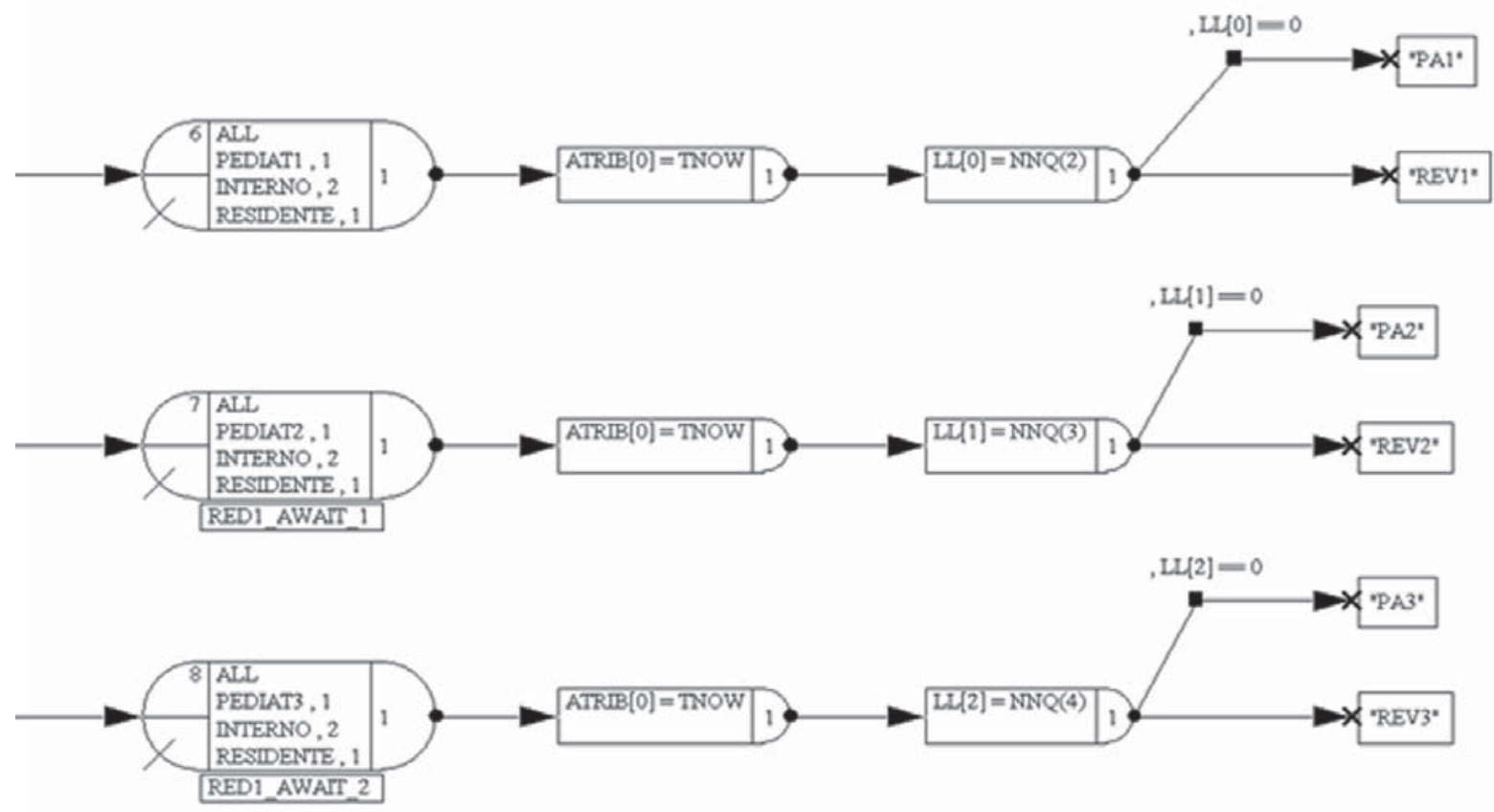

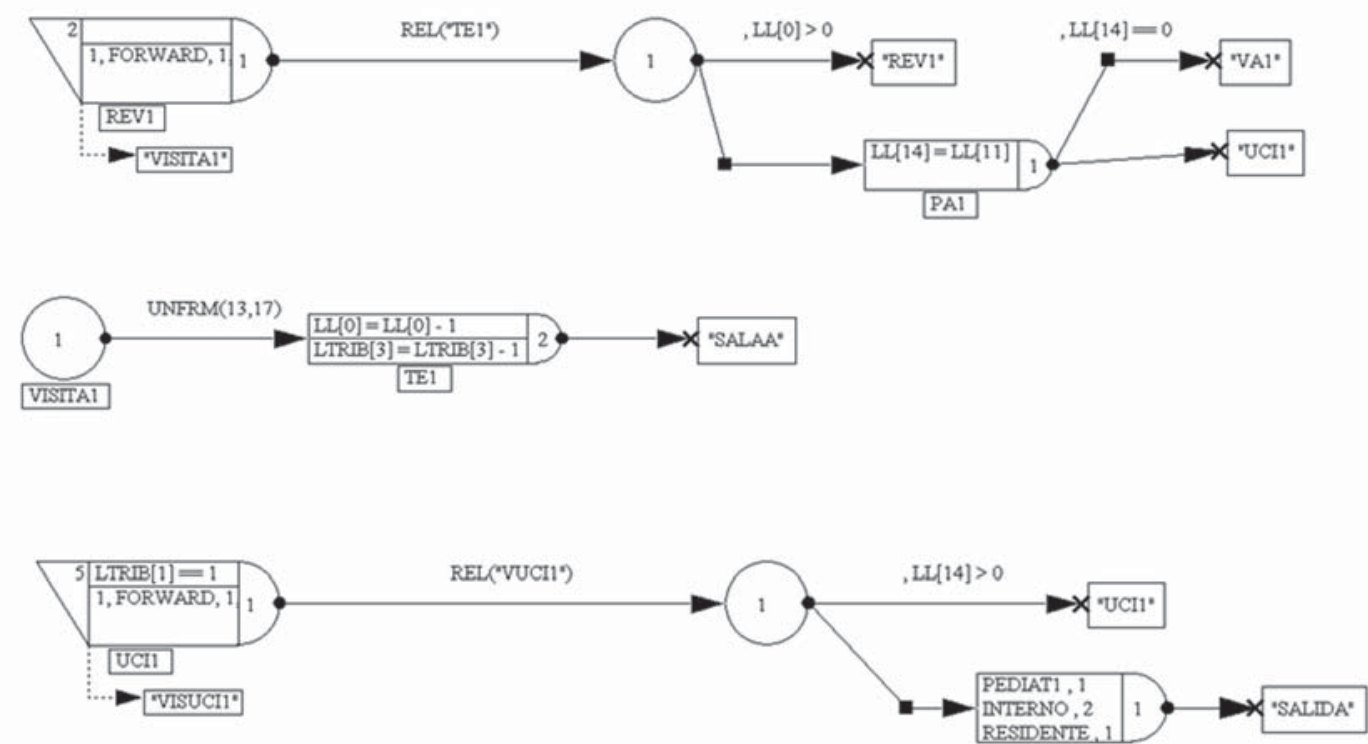

VA1
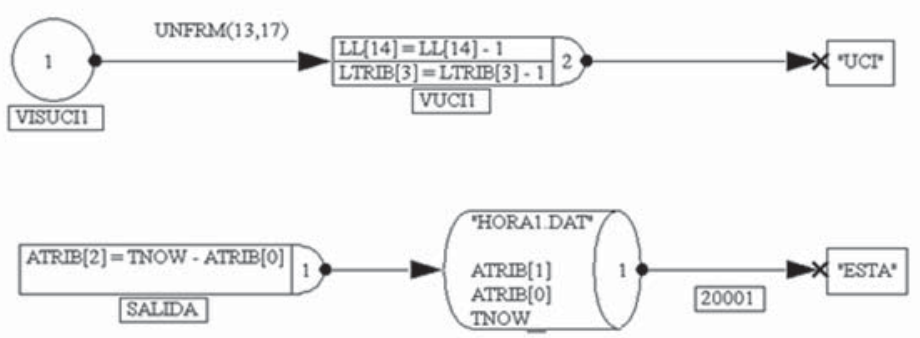

Figura 3. Visita diaria de los médicos: Trabajo típico del equipo de profesionales.

Elaboración propia.

\section{Resultados de la simulación y validación del modelo}

A continuación se mostrarán los resultados obtenidos de la simulación ejecutada, asimismo se validará el modelo propuesto.

\subsection{Medidas de validación}

Con el fin de realizar el análisis de resultados se han ejecutado treinta replicaciones. Para reducir la varianza del modelo, la cual se crea por razones de la aleatoriedad, se ha usado la técnica de reducción de varianza llamada secuencia común, amplia- 
mente utilizada en modelos de simulación. Para hacer la validación del modelo se tuvo que determinar algunas medidas de desempeño. Por este motivo se brindan determinados indicadores emitidos por la Dirección General de Salud Pública.

- Indicadores de desempeño

En este artículo se presentan algunos indicadores, los cuales se utilizan teniendo en cuenta que el H. N. Arzobispo Loayza es un hospital de tipo III:

- Para la productividad:

Indicador: Rendimiento hora médico

Fórmula de cálculo:

Número de atenciones

Número de horas médicas efectivas

Valor objetivo del indicador: Para los hospitales de tipo I es de 5,0; para los hospitales de tipo II es 4,5, y para los hospitales de tipo III e institutos especializados es de 4,0.

- Para la eficiencia:

Indicador: Intervalo de sustitución de cama

Fórmula de cálculo:

$\frac{\text { Días cama disponibles-Pacientes días }}{\text { Número de egresos }}$

Valor objetivo del indicador: Para los hospitales de tipo I, de tipo II, de tipo III e institutos especializados es 1,0.

- Indicador: Porcentaje de ocupación de cama

Fórmula de cálculo:

$$
\frac{\text { Total pacientes día }}{\text { Número de egresos }} * 100 \%
$$

Valor objetivo del indicador: Para los hospitales de tipo I, de tipo II, de tipo III e institutos especializados es $90 \%$.

- Indicador: Rendimiento cama

Fórmula de cálculo:

Número de egresos

Número de camas reales (promedio) 
Valor objetivo del indicador: Para los hospitales de tipo I es de 6,0; de tipo II es de 4,0; de tipo III e institutos especializados es 3,0.

El valor objetivo para los indicadores fue determinado por la actual administración de salud.

\subsection{Análisis de los resultados}

En el proceso de simulación, al terminar cada replicación, se extrajeron los resultados de las variables a archivos externos y haciendo uso de Minitab se obtuvieron los intervalos de confianza que se muestran en la siguiente tabla:

\begin{tabular}{|cccccc|}
\hline Variable & N & Mean & StDev & SE Mean & $95 \%$ CI \\
REND_HORA_MED & 30 & 3,51992 & 0,39186 & 0,07154 & $(3,37359 ; 3,66624)$ \\
INT_SUS_CAMA_1 & 30 & 1,15766 & 0,83171 & 0,15185 & $(0,84709 ; 1,46822)$ \\
PORC_UTIL_CAMA & 30 & 65,0769 & 17,7488 & 3,2405 & $(58,4494 ; 71,7044)$ \\
REN_CAM & 30 & 0,135385 & 0,037336 & 0,006817 & $(\odot, 121443 ; 0,149326)$ \\
\hline
\end{tabular}

Tabla 3. Estadística de los resultados de la simulación para treinta replicaciones.

Elaboración propia.

\subsection{Validación del modelo}

Para validar el modelo se compararon los valores obtenidos en la simulación, de los indicadores con respecto a los valores objetivos brindados por el Ministerio de Salud (Minsa) en la directiva Na - MINSA/OGPE - V.01, titulada "Evaluación de los planes operativos institucionales de las dependencias del Ministerio de Salud del 2002".

Actualmente, el hospital se encuentra redefiniendo sus procesos para poder alcanzar los valores pedidos, lo cual en cierta medida puede explicar las diferencias encontradas:

a) Para el indicador Rendimiento hora médico de la productividad, el valor dado por el Minsa es de 4,0 y el resultado de la simulación es de 3,51.

b) Para el indicador Intervalo de sustitución de cama para la eficiencia, el valor dado por el Minsa es de 1,0 y el resultado de la simulación es de 1,15.

c) Para el indicador Porcentaje de ocupación de cama para la eficiencia, el valor dado por el Ministerio es de $90 \%$ y el resultado de la simulación es de $65 \%$.

d) Para el indicador Rendimiento cama para la eficiencia, el valor dado por el Minsa es de 3,0 y el resultado de la simulación es de 0,13. 
Según la administración del H. N. Arzobispo Loayza estos valores son aceptables, conclusión a la que llegó la administración luego de revisar documentos internos de dicho nosocomio.

\section{Conclusiones y recomendaciones}

\subsection{Conclusiones}

a) El uso de la simulación puede determinar una nueva forma de administrar los recursos de un hospital, es decir apoyar en su administración hospitalaria.

b) La ejecución de la simulación propuesta permite estimar muchos de los indicadores de la gestión de un hospital.

c) Con un modelo que simule el proceso de atención a los pacientes, el cálculo de la cantidad de recursos necesarios para ello es mucho más eficiente y eficaz, por lo que el tiempo en la administración de estos recursos se reduce y su costeo se hace más sencillo.

d) La reducción de los tiempos en la gestión de los recursos reduce los costos de las prestaciones.

e) Conociendo el verdadero uso de los recursos hospitalarios se puede determinar un empleo más eficiente y eficaz de estos.

f) El modelo de simulación es amigable en su manejo, lo que permite una rápida adecuación en caso de existir un rediseño en los procesos inmersos en la gestión hospitalaria.

\subsection{Recomendaciones}

a) Procurar generar grupos de diagnóstico relacionados para el Perú, ya que existe diferencia entre la realidad sanitaria en Estados Unidos y la de nuestro país.

b) Si se desea obtener otros indicadores de la gestión hospitalaria, lo primero que debe hacerse es determinar su forma de cálculo y agregarse al modelo en la sección de estadísticas.

c) Para usar este modelo como herramienta de gestión se deberá realizar lo siguiente:

i. Desarrollar los procesos para los demás DRG inmersos en la gestión hospitalaria respectiva.

ii. Preparar una interfaz de ingreso de datos y otra de salida de datos para el uso más eficaz y eficiente por parte de los encargados de la administración de los recursos hospitalarios. 
Alberto Cossa Cabanillas

\section{Bibliografía}

Hospital Nacional Arzobispo Loayza (2000). Memoria anual 2000. Lima: Hospital Nacional Arzobispo Loayza, Oficina Ejecutiva de Planeamiento Estratégico.

Ministerio de Salud (2002). Evaluación del plan operativo anual 2002. Lima: Ministerio de Salud, Oficina Ejecutiva de Planeamiento Estratégico.

Ministerio de Salud (2001). Manual de indicadores de salud hospitalarios. Lima: Ministerio de Salud, Oficina General de Epidemiología. 